\title{
Niemand, Stephan: Alltagsumbrüche und Medienhandeln. Eine qualitative Panelstudie zum Wandel der Mediennutzung in Übergangsphasen.
}

\author{
Wiesbaden: Springer VS 2020. 264 Seiten. Preis: $€ 49,99$
}

\author{
Elisa Pollack
}

Angenommen: 6. März 2021 / Online publiziert: 23. März 2021

(C) Der/die Autor(en) 2021

Dass Medien- und Alltagshandeln eng miteinander verwoben sind, ist längst ein Gemeinplatz der Kommunikationswissenschaft. Wie aber verschiedene Alltagsumbrüche - eine Trennung, ein Umzug oder der Tod der PartnerIn - konkret die Nutzung unterschiedlicher Medien beeinflussen, wurde bisher nur unzureichend untersucht. Diesen ,blinden Fleck in der kommunikationswissenschaftlichen Rezeptionsforschung“ (S. 2) möchte Stephan Niemand in seiner Dissertation überwinden. Hierfür wählt er eine qualitative Herangehensweise und setzt unmittelbar an den MediennutzerInnen an. Die Arbeit wurde mit dem Dissertationspreis der WWU Münster sowie dem Dissertationspreis der Fachgruppe Soziologie der Medienkommunikation der DGPuK ausgezeichnet.

Im ersten Teil der Arbeit entwickelt der Autor einen gut begründeten theoretischen Rahmen, der das Verhältnis zwischen Alltag und Medienhandeln konzeptualisiert. Die Ansätze von Mediatisierung und Domestizierung werden gewinnbringend mit dem soziologischen Konzept der alltäglichen Lebensführung sowie dem der Transitionsprozesse verknüpft. Dabei erfährt die in der Kommunikationswissenschaft häufig zum Einsatz kommende, jedoch selten spezifizierte Kategorie des Alltags eine begriffliche Schärfung. Aus den theoretischen Vorannahmen schlussfolgert Niemand fünf Prinzipien, die seine empirische Analyse anleiten. Die hier aufgestellten Forderungen nach Ganzheitlichkeit, Kontextorientierung, Prozessorientierung, Offenheit der Entwicklungsrichtung und Nutzerorientierung können über die vorliegende Untersuchung hinaus als allgemeine Orientierungspunkte für künftige Rezeptionsstudien mit ähnlichem Erkenntnisinteresse gelten.

E. Pollack $(\square)$

Institut für Publizistik- und Kommunikationswissenschaft, Freie Universität Berlin,

Otto-von-Simson-Straße 3, 14195 Berlin, Deutschland

E-Mail: elisa.pollack@fu-berlin.de 
Im zweiten, empirischen Teil differenziert Niemand seine Fragestellungen weiter aus und schildert sein methodisches Vorgehen. Zunächst wird der Entstehungskontext der Forschungsdaten offengelegt. Diese wurden im Rahmen der DFG-geförderten Panelstudie „Das mediatisierte Zuhause“ erhoben. Dort wurden die Interaktionsdynamiken in Paarbeziehungen zu zentralen Kontextfaktoren erklärt, die zum Verständnis des Medienhandelns im Alltag berücksichtigt werden müssen. Methodisch wurde daher der in diesem Kontext durchaus überzeugende Ansatz des ethnografisch-orientierten Paar-Interviews verfolgt. An vier Zeitpunkten zwischen 2008 und 2016 wurden 25 Paarhaushalte besucht und zu deren Lebensführung und Mediennutzungsverhalten befragt. Dabei wurden verschiedene Alltagsumbrüche ermittelt. Am häufigsten im Sample vertreten waren die Elternschaft, der Auszug der Kinder, die Trennung und der Umzug. Für die Haushalte, in denen sich Alltagsumbrüche ereigneten, fertigt Niemand ,medienethnografische Porträts“ (S. 104) an, deren thematische Struktur sich an einem zuvor entwickelten Themen- und Leitfragenkatalog orientiert. Im Anschluss an die Einzelfallbeschreibung, in der schlüssig dargelegt wird, wie die verschiedenen Alltagsumbrüche je einen Wandel der häuslichen Mediennutzung anstoßen, erfolgt eine fallvergleichende Analyse. Diese mündet in eine zusammenfassende Darstellung acht ,,alltagsspezifischer Antriebsfaktoren für Dynamik im Medienrepertoire“ (S. 217), die auf einer zuvor angestellten Differenzierung der Lebensführung in verschiedene Dimensionen (Zeit, Inhalte, Räumliches, Soziales, Materielles, Sinn, Emotionen, Körper) basiert. Positiv hervorgehoben werden soll an dieser Stelle die durchgängig gute Nachvollziehbarkeit des Vorgehens, die hier durch eine detaillierte Beschreibung des Auswertungsprozesses sowie durch die Arbeit mit zahlreichen Interviewzitaten erreicht wird.

Der dritte und letzte Teil der Arbeit beginnt mit einer Reflexion der Stärken und Schwächen der Untersuchung. Daran anschließend wird durch die Zusammenführung von Theorie und Empirie ein theoretisches Konzept zur Verwobenheit von Alltagsumbrüchen und Medienhandeln entwickelt. Es entsteht ein heuristisches Modell, mit dem die Prozesse, die zu einem Wandel der Mediennutzung führen, systematisch erfasst werden können. Dabei wird offensichtlich, dass sich Medienhandeln in Abhängigkeit von Bedürfnissen und Daseinsthematiken, Zwängen und Erwartungen sowie von Entfaltungschancen vollzieht. Darauf aufbauend führt Niemand den Begriff der ,mediatisierten Lebensführung“ (S. 242) ein. Mit diesem Begriff lässt sich die Anbindung von Mediennutzung an konkrete Lebenssituationen adäquater fassen als mit dem Begriff des Medienhandelns. In seiner Beschreibung des Zusammenhangs von Alltagsfaktoren und Mediennutzung geht Niemand deutlich über die bekannten Annahmen des Uses-and-Gratifications-Ansatzes hinaus. Er illustriert anschaulich wie eng Medienhandeln und individuelle Lebensführung miteinander verknüpft sind und ist so darüber hinaus in der Lage technikdeterministische Annahmen zurückzuweisen, die vor allem medientechnologische Veränderungen als wesentliche Ursache für einen Wandel der Mediennutzung ausmachen.

Stephan Niemand argumentiert stets schlüssig und immer im Hinblick auf seine konkrete Fragestellung. So ist eine kurzweilige Monographie entstanden, deren Gehalt auch durch Fachfremde leicht erschlossen werden kann. Die Arbeit überzeugt sowohl in ihrer theoretischen Konzeption als auch ihrer methodischen Umsetzung und vermag es, einen Beitrag zur kommunikationswissenschaftlichen Theoriebil- 
dung zu leisten. Fokussiert sich der Autor zwar entsprechend seines Erkenntnisinteresses auf Umbrüche und Entwicklungen, die auf der Mikroebene angesiedelt sind, so kann seine Systematik auch als Werkzeug für künftige Untersuchungen fungieren, die sich für die Auswirkungen gesamtgesellschaftlicher Wandlungsprozesse auf individuelle Mediennutzung interessieren.

Funding Open Access funding enabled and organized by Projekt DEAL.

Open Access Dieser Artikel wird unter der Creative Commons Namensnennung 4.0 International Lizenz veröffentlicht, welche die Nutzung, Vervielfältigung, Bearbeitung, Verbreitung und Wiedergabe in jeglichem Medium und Format erlaubt, sofern Sie den/die ursprünglichen Autor(en) und die Quelle ordnungsgemäß nennen, einen Link zur Creative Commons Lizenz beifügen und angeben, ob Änderungen vorgenommen wurden.

Die in diesem Artikel enthaltenen Bilder und sonstiges Drittmaterial unterliegen ebenfalls der genannten Creative Commons Lizenz, sofern sich aus der Abbildungslegende nichts anderes ergibt. Sofern das betreffende Material nicht unter der genannten Creative Commons Lizenz steht und die betreffende Handlung nicht nach gesetzlichen Vorschriften erlaubt ist, ist für die oben aufgeführten Weiterverwendungen des Materials die Einwilligung des jeweiligen Rechteinhabers einzuholen.

Weitere Details zur Lizenz entnehmen Sie bitte der Lizenzinformation auf http://creativecommons.org/ licenses/by/4.0/deed.de.

Elisa Pollack ist wissenschaftliche Mitarbeiterin am Institut für Publizistik- und Kommunikationswissenschaft der Freien Universität Berlin. 